\title{
A Light-Scattering Study of the Scattering Matrix Elements of Arizona Road Dust
}

Yuli Wang, Amitabha Chakrabarti, Christopher M. Sorensen*

Department of Physics, Kansas State University, Manhattan KS 66506 USA

* Corresponding author at: Department of Physics, Kansas State University, Manhattan KS 66506 USA. E-mail address: sor@phys.ksu.edu

Abstract. We report measurements of the light scattering matrix elements of Arizona Road Dust which has irregular shapes. Our experimental apparatus used photodiode arrays to detect scattered light simultaneously at many angles including small angles that are necessary for accurate characterization of large particles. The setup was calibrated with single slit diffraction and water droplet scattering. Guinier analysis yielded the dust particle size. Q-space analysis of the dust scattering yielded a comprehensive description of scattering in terms of power laws with quantifiable exponents.

Keywords:

Light scattering

Arizona Road Dust

Q-space analysis

Guinier analysis 


\section{Introduction.}

Scattered light from spherical particles has been studied for more than 100 years and is well understood [1, 2]. However, light scattering from irregularly shaped particles remains a puzzle. Not only does scattering from irregularly shaped particles hold inherent intellectual interest, such scattering plays an important role in affecting the global radiation budget. In this paper we describe light scattering experiments conducted on Arizona Road Dust (AZRD) which is a canonical example of irregularly shaped particles similar to many other dusts that can be found in the atmosphere. Curtis et al. has studied AZRD and the scattering angles were $17^{\circ}$ to $172^{\circ}$ [3].The Amsterdam group has studied a number of aerosol particles with an apparatus that allowed for measurements from $3^{\circ}$ to $177^{\circ}[4,5]$. Our apparatus has some advantages over other similar instruments such that it detects light at many angles simultaneously. Our apparatus was built to detect from a small angle of $0.32^{\circ}$ to $157^{\circ}$. This factor of 10 decrease in the smallest forward angle is very important for detecting the beginning of scattering angle functionality in the small angle limit, especially for large particles, and to determine particle sizes. In this paper, we present our apparatus which can detect scattered light at many angles simultaneously, the calibration methods for the whole system, and the results from three different sizes of AZRD. Our work also differs from previous work in that Q-space analysis [6-8] is applied to our experimental results. Instead of plotting scattered intensity vs. the scattering angle $\theta$ on a linear scale, Q-space analysis graphs scattered intensity vs. the magnitude of the scattering wave vector $q$ on a log-log scale. The scattering wave vector $q$ has a magnitude of $2 k \sin (\theta / 2)$, where $k=2 \pi / \lambda$. We find that Q-space analysis reveals power law functionalities with quantifiable exponents. Moreover, Q-space analysis with Guinier analysis (discussed in section 5) indicates particle sizes. The importance of our work is the validation of our novel experimental apparatus, demonstration of the utility of the Q-space analysis, and describing light scattering from aerosols of irregularly shaped particles.

\section{The scattering matrix.}

The optical scattering information about a given particle is completely described by the 4 by 4 scattering matrix. When the particles are randomly oriented and have a plane of symmetry, the scattering matrix has eight nonzero elements:

$$
\left(\begin{array}{cccc}
S_{11} & S_{12} & 0 & 0 \\
S_{21} & S_{22} & 0 & 0 \\
0 & 0 & S_{33} & S_{34} \\
0 & 0 & S_{43} & S_{44}
\end{array}\right),
$$

where $S_{21}=S_{12}$ and $S_{43}=-S_{34}[9,10]$. Thus, six of the matrix elements are independent. This matrix applies to an ensemble of randomly oriented AZRD particles [11].

The intensity and polarization of a beam of light can be specified by a Stokes vector:

$$
\left(\begin{array}{llll}
I & Q & U & V
\end{array}\right)^{T} \text {, }
$$

where $T$ is the transpose operator. I represents the total intensity, $Q$ represents the difference between the horizontally and vertically polarized intensities, $U$ represents the difference between the $+45^{\circ}$ and $45^{\circ}$ polarized intensities, and $V$ represents the difference between the right-handed and left-handed polarized intensities $[9,10]$.

The Stokes vector of the scattered light is obtained by multiplying the incident light Stokes vector by the 4 by 4 scattering matrix: 


$$
\left(\begin{array}{cccc}
S_{11} & S_{12} & 0 & 0 \\
S_{12} & S_{22} & 0 & 0 \\
0 & 0 & S_{33} & S_{34} \\
0 & 0 & -S_{34} & S_{44}
\end{array}\right) \cdot\left(\begin{array}{c}
I_{i n c} \\
Q_{i n c} \\
U_{i n c} \\
V_{i n c}
\end{array}\right)=\left(\begin{array}{c}
I_{s c a} \\
Q_{s c a} \\
U_{s c a} \\
V_{s c a}
\end{array}\right)
$$

By sending certain polarized light incident upon the particulate system and then detecting the difference between certain polarized scattered intensities, one can measure all six matrix elements $[9,10]$.

\section{Experiment.}

3.1. Experimental Apparatus. Figure 1 is a schematic diagram of the experimental apparatus. The laser beam with $\lambda=532 \mathrm{~nm}$ passed through a linear polarizer or a wave plate on its way toward the aerosol from which it scattered. A linear polarizer or a wave plate was placed between the aerosol particles and the detector for both forward and side scattering. The forward scattering detection followed a design by Ferri [12] with a beam stop placed at the focal point of a Fourier lens to block the light directly from the laser. The scattered light was collected by a lens that imaged the Fourier plane onto the detector. For the side scattering, a custom elliptical mirror (Optiforms) collected a wide range of angles. The intersection of the incident light and the aerosol was at one focal point of the elliptical mirror, and an iris was placed at the other focal point. This scattered light was then collimated by a lens. Two 16 channel photodiode arrays (Hamamatsu S8558) were used as detectors at a total of 31 angles. One channel of the side detector was sacrificed as a monitor. The two detectors were connected to a data acquisition box connected to a computer. The use of photodiode arrays to detect light at many angles simultaneously allows for quick and efficient procurement of data, eliminates problems regarding aerosol stability, and makes detection at small angles easier.

Based on the information of the scattered light Stokes vector and the scattering matrix described in section 2 , one can measure all six matrix elements by manipulating the polarizers and wave plates. To measure S11, we send circularly polarized light (produced by a $\lambda / 4$ wave plate after the vertically polarized laser with fast axis $45^{\circ}$ from vertical) and measure the intensity of the scattered light. To measure $-\mathrm{S} 12$, send circularly polarized light and measure the difference between the vertically and horizontally polarized scattered intensities with a linear polarizer. S22 is the difference between two situations: first send horizontally polarized light ( $\lambda / 2$ wave plate with fast axis $45^{\circ}$ from vertical) and measure the difference between horizontally and vertically polarized intensities, then send vertically polarized light $(\lambda / 2$ wave plate with fast axis vertical) and measure the difference between horizontally and vertically polarized intensities. So on and so forth, to measure S33/S11, send 45 degree polarized light and measure the difference between the $+45^{\circ}$ and $-45^{\circ}$ polarized intensities. To measure S34/S11, send circularly polarized light and measure the difference between the $+45^{\circ}$ and $-45^{\circ}$ polarized intensities. To measure S44/S11, send circularly polarized light and measure the difference between the right-handed and left-handed polarized intensities. 




Figure 1. A schematic diagram of the experimental apparatus.

3.2. Calibrations. A $10 \mu \mathrm{m}$ single slit was used to calibrate the angles of the 16 channels in the forward direction. Figure 2 shows that the experimental and theoretical patterns of single slit diffraction match well under Q-space analysis except for the theoretical minima because the photodiodes have finite angular width and are not sensitive enough to detect very low intensity. Q-space analysis reveals a power law envelop with an exponent of -2 which is the expected value of $-\left(2 D_{m}-D_{s}\right)$, where $D_{m}$ is the mass dimension and $D_{S}$ is the surface dimension of the single slit [8] ( 1 and 0 , respectively). The angles of the 15 channels at the side direction are determined from geometrical calculation. Each value labeled in Figure 1 corresponds to the center of each channel. Since each channel has its width, each angle also has its spread in angle. The corresponding error bars will be shown in Figures 2, 5, 6, 7, and 8.



Figure 2. Single slit diffraction experimental and theoretical patterns for the forward direction. Intensity (arbitrary units) is plotted vs. q $\left(\mathrm{cm}^{-1}\right)$. The -2 slope line indicates the power law of the theoretical curve. 
We calibrated the whole system by measuring S11 of water droplets produced from an atomizer, as shown as diamonds in Figure 3a. A multiplication factor was necessary to connect the 15 side-channels to the 16 front-channels. Furthermore, the first channel on the side detector was found to give a low signal which means another multiplication factor was needed for that channel. Figure 4 shows the setup used to determine those two calibration factors. We placed two mirrors to direct the laser beam such that $0^{\circ}$ scattering angle was offset by $6.48^{\circ}$. Thus, the side scattering detector included an angle -8.82 ? - which was in the angle range of the front scattering detector.


Figure 3. A calibration of the whole system by measuring S11 of water droplets using setup 1 and 4 . The S11 (arbitrary units) is plotted vs. the scattering angle.

Scattering Angles (degrees)

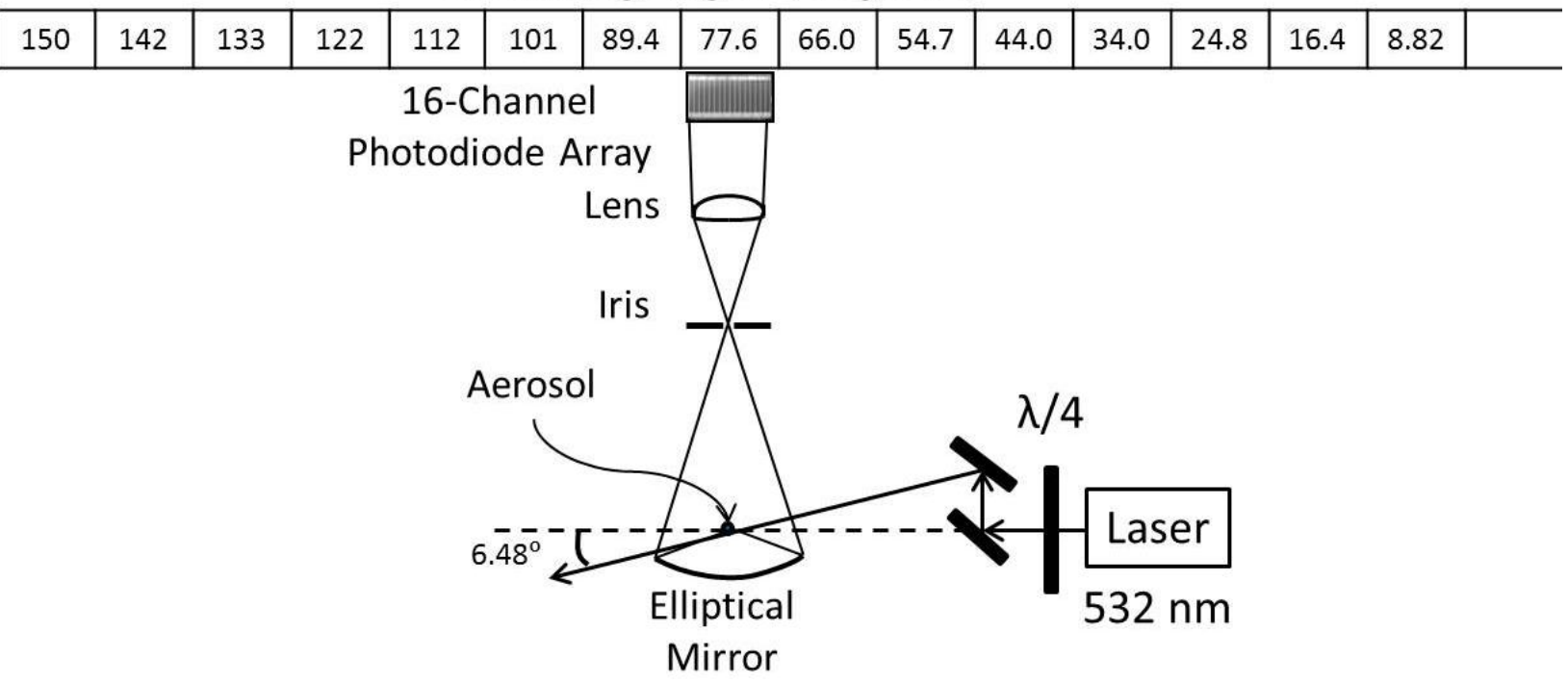

Figure 4. A schematic of the calibration of the whole system.

The setup in Figure 4 was used to measure S11 of water droplets and this S11 is shown as squares in Figure 3a. By comparing the side scattering $\left(>12^{\circ}\right)$ of both setups, the calibration factor to connect the side scattering to the front scattering was determined. After applying this factor to the side scattering, as shown in Figure $3 \mathrm{~b}$, the front scattering $\left(<10^{\circ}\right)$ of both schemes were compared to determine the second calibration factor that corrected the single low channel. From Figure $3 b$, the 
channels 10 and 11 (open circles) on the side detector had the same problem as the first channel. Thus the same correction method applied to channel 1 was applied to channel 10 and then channel 11 . Figure $3 c$ shows the final graph after all the corrections.

Figure 5 shows the experimental data of S11 (solid circles) for water droplets under the Q-space analysis. From now on, for convenience, we normalize S11 at the smallest q or angle. S11 experimental data shows two lines which have power laws of $q^{-2}$ and $q^{-4}$. Here, we introduce $P(R)$ which is the unnormalized log-normal size distribution given by

$$
P(R)=\exp \left(-\ln ^{2}\left(R / R_{0}\right) / 2 \ln ^{2} \sigma\right),
$$

where $R_{0}$ is the most probable radius and $\sigma$ is the geometric width of the distribution [13]. Fitting our experimental data for S11 with the theoretical Mie code [2] (solid line) averaged over the size distribution yielded a best fit weighted mean radius of our water droplets to be $1.1 \mu \mathrm{m}$ with a geometric width of 1.5 for a log-normal distribution. A multiplication factor was applied to the original theory curve to make the theoretical S11 normalized at the smallest q. The theory shows an obvious dip at $q \approx 30000 \mathrm{~cm}^{-1}$ but our experiment does not. We speculate that the lack of the dip of our experiment is possibly due to the non-sphericity of our water droplets based on our theoretical calculations for slightly aspherical drops that show lessened dips.

To verify that the experiment was carried out in the single scattering limit, a power meter was placed after the aerosol to detect the power before and after the aerosol was turned on. $I=I_{0} e^{-<s>}$ where $I$ is the power for the aerosol on, $I_{0}$ is the power for the aerosol off, and $\langle s\rangle$ is the average number of scattering events [14]. In our case, the power for the aerosol on was $92.3 \%$ of the power for the aerosol off, which means the average number of scattering events $\langle s>$ was 0.08 . Since multiple scattering events are distributed by a Poisson distribution [14], this low value indicates the experiment was under the single scattering limit.

Figure 6 shows the six matrix elements vs. the scattering angle $\theta$ for the water droplets. By repeating the experiments, we determined that the uncertainties of the matrix elements are within the size of the solid circles. The uncertainty of each angle is due to the width of each channel of the detectors. Compared to Q-space analysis of Figure 5, Figure 6a for the scattered intensity S11 does not have any quantifiable exponent to describe the trend. Another observation strongly supporting the nonsphericity of our water droplets is that S22/S11 decreases from unity as the scattering angle increases as shown in Figure 6c. Our water droplets experimental data agreed well with the Amsterdam group's water droplets data provided on their website. Theoretical work has shown that even a few percent of deviation from spheres can cause a significant change in the matrix elements. A reason for the nonsphericity of our water droplets is that the water droplets coming out of the atomizer might oscillate with certain modes. Future work will investigate these possibilities further. 




Figure 5. Experimental water droplet S11 data compared to Mie theory for perfect, polydisperse spheres with weighted mean radius of $1.1 \mu \mathrm{m}$ and a geometric width of 1.5 for a log-normal distribution. S11 is normalized at the smallest $\mathrm{q}$.
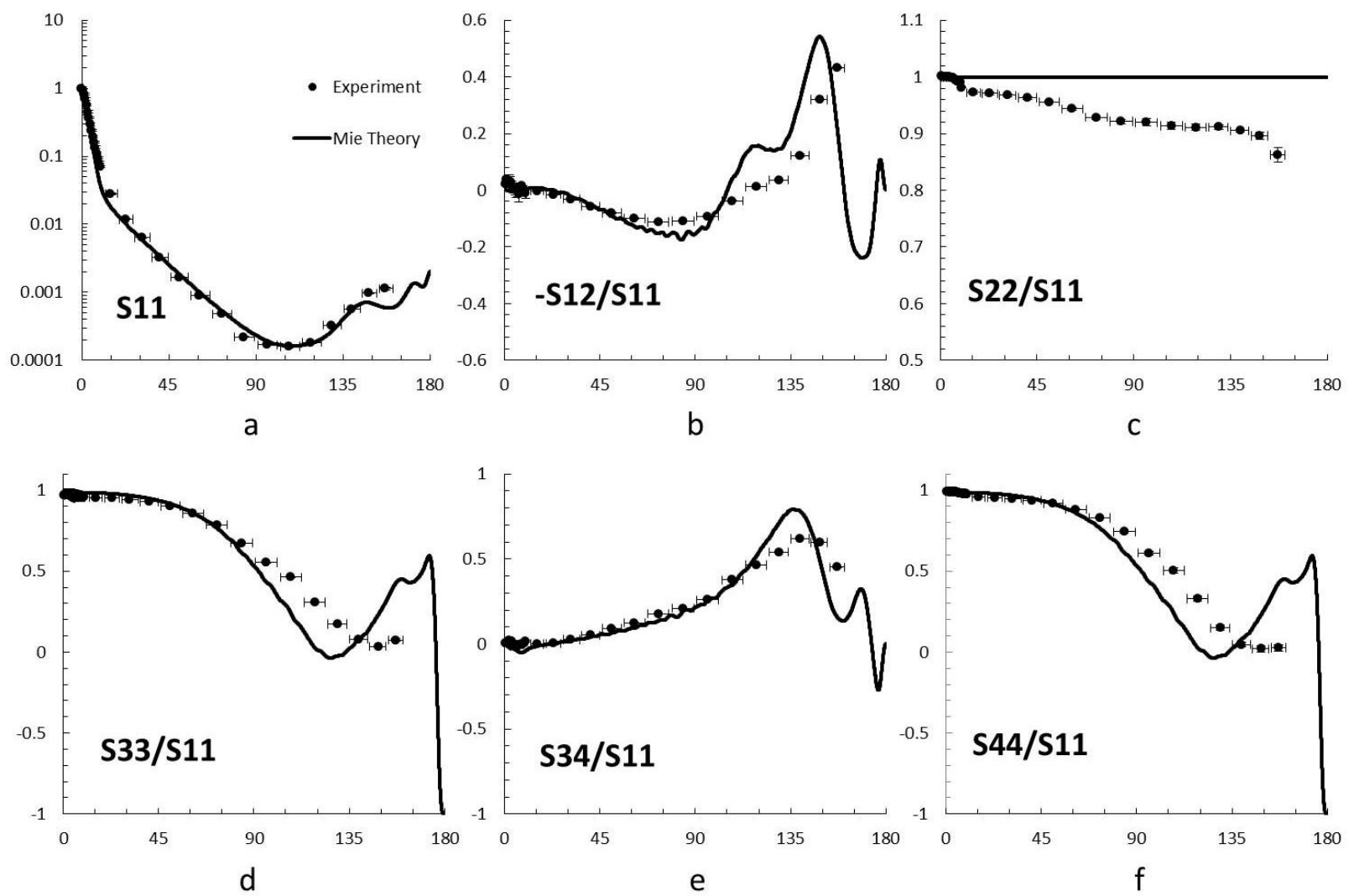

Figure 6. The scattering matrix elements vs. the scattering angle $\theta$ for water droplets produced from an atomizer compared to Mie theory. S11 is normalized at the smallest angle. 
3.3. Dust Aerosol Generation. We used a dust generator to aerosolize the dust. The dust generator had a three inch in diameter and three inch tall cylindrical chamber into which the dust was loaded. The bottom of the chamber had a stir bar which spun at 200 revolutions per minute. Simultaneously, oxygen blew in from the bottom of the chamber. The outgoing aerosolized dust particles were directed to the scattering volume by vinyl tubing. The dust aerosol was emitted vertically from a $3 \mathrm{~mm}$ ID brass tube 10 $\mathrm{mm}$ below the scattering volume.

\section{Results.}

We present the results for the scattering matrix elements of AZRD (Powder Technology Inc.) which is a type of standard dust used in filter testing. Figure 7 shows the Q-space analysis on the three sizes of AZRD (Ultrafine, Fine, and Medium). The exponents of the power laws of the Q-space analysis on the Ultrafine, Fine and Medium AZRD samples are $-2.23,-2.17$, and -2.12 , respectively, all with uncertainties of \pm 0.05 , hence nearly equivalent. Figure 8 shows the six matrix elements vs. $\theta$ for the three sizes of AZRD. The smallest angle of our forward scattering is $0.32^{\circ}$ which is a factor of 10 smaller than the smallest angle of all previous work on irregularly shaped particles [3-5]. This allows us to detect both the Guinier regime and constant intensity forward scattering lobe which mark the end of the angular functionality in the small angle limit. Furthermore, with the Guinier regime, one can characterize the sizes of the large particles [15], which will be discussed in section 5 . The forward scattering $\left(0.32^{\circ}\right.$ to $9.89^{\circ}$ ) for all matrix elements except for S11 is enlarged in an inset in Figures $8 b$ to $\mathrm{f}$. In Figures $8 \mathrm{~b}$ and e for $-\mathrm{S} 12 / \mathrm{S} 11$ and S34/S11, respectively, the forward scattering is fluctuating around 0 without any functionality with considerable variation. This variation is due to two nearly equal intensities subtracted from each other to obtain those matrix elements. Ideally, the forward scattering of $-\mathrm{S} 12 / \mathrm{S} 11$ and S34/S11 would align to 0 , which is consistent with the data.



Figure 7. The Q-space analysis on the Ultrafine, Fine and Medium AZRD samples. S11 of three ARZD samples are normalized at the smallest q. 



Figure 8. The scattering matrix elements vs. $\theta$ for three sizes of AZRD. S11 of three ARZD samples are normalized at the smallest angle.

\section{DATA ANALYSIS AND DISCUSSION.}

The scattering matrix elements of AZRD, which has irregular shapes, behave differently from the scattering matrix elements of the water droplets - "quasi-spherical" particles. For non-spherical particles, S22/S11 is no longer unity for all scattering angles. Our work finds the six matrix elements of AZRD agree very well with the summary work done by the Amsterdam group on many different types of irregular shaped particles [4].

Based on the Q-space analysis, regardless of the shape of the particle, one can determine the size of the particle by doing Guinier analysis. For a sphere with radius of gyration $R_{g}$ the Guinier equation is [15]

$$
I(q)=I(0)\left(1-q^{2} R_{g}^{2} / 3\right)
$$

Guinier analysis is applicable when $q R_{g}<1$, although one can often exceed this limit [15]. At the limit where $q R_{g}=1, I(q)$ is $2 / 3$ of $I(0)$. In Figure 5 , at $2 / 3$ of the maximum intensity, the corresponding $q$ indicates that $R_{g}=2.4 \mu \mathrm{m}$. When $q R_{g}$ is very small, Eq. (3) can be rewritten as

$$
I(0) / I(q)=1+q^{2} R_{g}^{2} / 3 .
$$

Thus the slope of $I(0) / I(q)$ versus $q^{2}$ is $R_{g}^{2} / 3$, and the data are plotted in this manner in Figure 9 to find a measured radius of gyration of $R_{g}=2.5 \mu \mathrm{m}$. On average, the measured radius of gyration is $2.45 \mu \mathrm{m}$. By multiplying $R_{g}$ by $\sqrt{5 / 3}$, the measured geometric radius is $R=3.2 \mu \mathrm{m}$. The measured radius is a significant discrepancy from $1.1 \mu \mathrm{m}$ obtained from the Mie code discussed in section 3 . 




Figure 9. Guinier analysis of water droplet.

One reason for the measured radius being bigger than the true radius is that particles have a size distribution and bigger particles scatter more light than the smaller particles. The light scattered by bigger particles affects the average more.

However, there are more subtle effects on the Guinier analysis for refractive particles which are identified as having a phase shift parameter $\rho=2 k R|m-1|$, where $m$ is the relative refractive index, greater than one. These effects were first identified some time ago [16]; here we apply them for the first time in what we will call the Extended Guinier Analysis. One must realize that the radius of gyration determined from the Guinier analysis, $R_{g, G}$, is not equal to the true radius of gyration, $R_{g}$, when $\rho>1$. Figure 10 shows the ratio $R_{g, G} / R_{g}$ vs. $\rho$ for monodisperse water droplets [16].

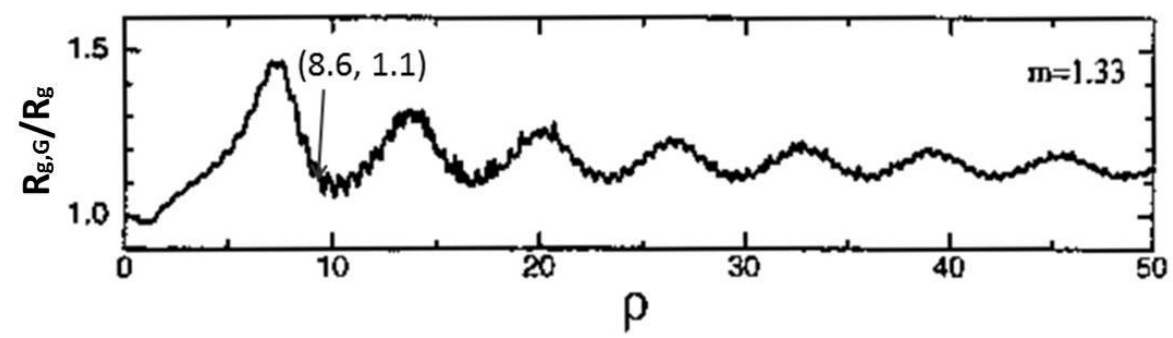

Figure 10. Monodisperse water droplet $R_{g, G} / R_{g}$ vs. $\rho$ [16]. (Adapted from Sorensen CM, Shi D, "Guinier analysis for homogeneous dielectric spheres of arbitrary size")

When the true radius is $1.1 \mu m\left(R_{g}=0.85\right), \rho=8.6$. According to Figure $10, R_{g, G} / R_{g}=1.1$, which means $R_{g, G}=0.94 \mu \mathrm{m}$. To determine the measured radius of gyration $R_{g, \text { meas }}$, integrating the size distribution into Guinier analysis Eq. (4),

$$
I(0) / I(q)=1+\left(q^{2} / 3\right)\left(\int R_{g}{ }^{4} R_{g}{ }^{2} P(R) d R_{g} / \int R_{g}{ }^{4} P(R) d R_{g}\right)=1+\left(q^{2} / 3\right) R_{g, \text { meas }}^{2},
$$

where $R_{g}$ is a variable and $R_{g}^{4}$ is light scattering weighting factor for forward scattering for large refractive particles [13]. Substitution of Eq. (2) with $R=R_{g} R_{0}=R_{g, G}=0.94 \mu m$ and $\sigma=1.5$ into Eq. 
(5) and solving the integral for $R_{g \text {,meas }}^{2}$ yields the measured radius of gyration $R_{g, \text { meas }}=2.5 \mu \mathrm{m}$. This is equivalent to a the measured geometric radius of $R=3.2 \mu \mathrm{m}$. Thus consistency between the Extended Guinier Analysis and the Mie fit is achieved. In summary, we start from the Mie theory which indicates the true radius is $1.1 \mu \mathrm{m}$ (radius of gyration $0.85 \mu \mathrm{m}$ ) with a geometric width 1.5. Then we apply the Extended Guinier Analysis and find the measured radius of gyration should be $2.5 \mu \mathrm{m}$ which agrees with the radius of gyration determined experimentally.

Figure 7 shows that the Guinier regimes of the S11 plots occur at smaller q for dusts with increasing sizes as indicated by the supplier. This is as expected. Figure 11 shows Guinier analysis, $I(0) / I(q)$ versus $q^{2}$, for the three sizes of AZRD. The Ultrafine dust has several points for the Guinier analysis. From the slope the radius of gyration of ultrafine dust is $2.7 \mu \mathrm{m}$. For Fine dust and Medium dust, the data are more limited. We put two boundary slopes for each set of data. The fit lines (solid lines) with the smaller slope are the fits for the all the points in a set and the fit lines (dashed lines) with the larger slope only include data when $q R_{g}<1$, where the Guinier analysis is, in principle, applicable. According to the fit line slopes, the ranges of radius of gyration are between 3.5 and $5.5 \mu \mathrm{m}$ for Fine dust and between 6.4 and $9.7 \mu \mathrm{m}$ for Medium dust. Although the fits for Fine and Medium dust in the $q R_{g}<1$ range only include two points, the estimated sizes are close to the sizes the manufacturer claimed. According to Powder Technology Inc., the median size of Ultrafine dust particles is $4.5 \mu \mathrm{m}$ in diameter, the median size of Fine dust particles is $10 \mu \mathrm{m}$ in diameter, and the median size of Medium dust particles is $15 \mu \mathrm{m}$ in diameter. Figure 12 shows the images with $10 \mu \mathrm{m}$ scaling bars for three AZRD samples under a binocular compound optical microscope. The measured radii of gyration of Ultrafine, Fine, and Medium AZRD are $2.7 \mu \mathrm{m}, 5.5 \mu \mathrm{m}$, and $9.7 \mu \mathrm{m}$ respectively, which agree well with the images according to the scaling bars. In the future, we would like to replace the 16 channel photodiode array in the forward direction with a 512 channel array which would allow us to approach much smaller angles with higher angular resolution, and then we would be able to characterize the particles sizes more precisely. Until we achieve better data and determine the manner in which the Extended Guinier Analysis can be applied to irregularly shaped particles, further analysis of that sort is not realizable.

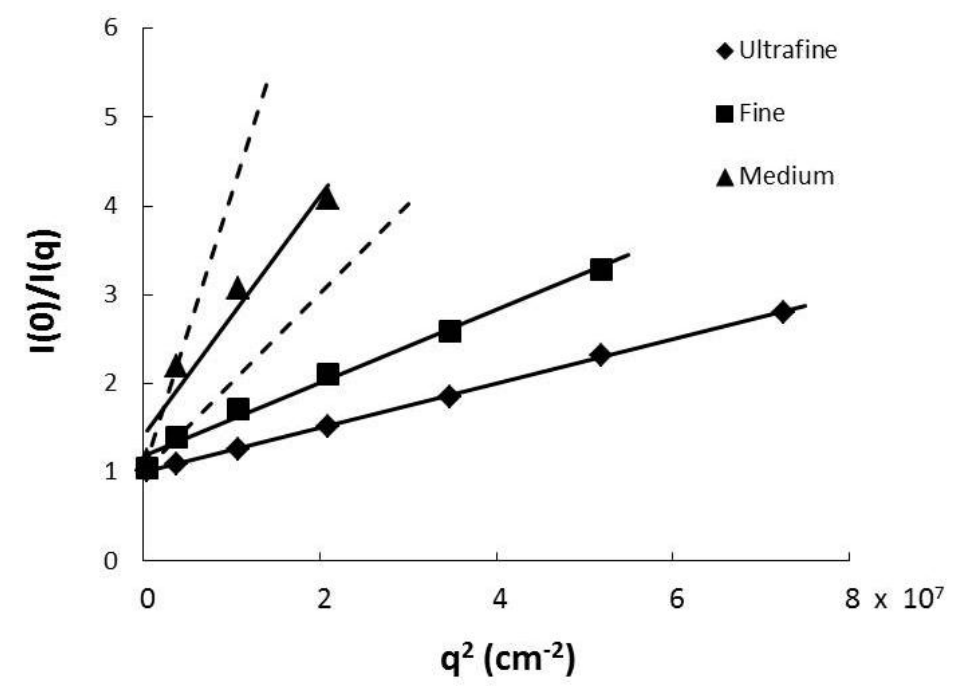

Figure 11. Guinier analysis of AZRD, Ultrafine, Fine and Medium. 



Figure 12. The images for three AZRD samples under an optical compound microscope. Each figure has a 10 micron scale bar in the lower left and a scale bar equal to the light scattering determined $2 \mathrm{Rg}$ in the upper right for comparison to the images. Note that light scattering is strongly affected by the largest particles in an ensemble.

Returning to Figure 5 we see that the Q-space analysis of the water droplet data for the scattered intensity, S11, shows power laws with exponents of -2 and -4 . These power laws lie in the region beyond the forward scattering lobe and the Guinier regime but before the backscattering regime where the scattering is enhanced. This confirms the same patterns seen by Q-space analysis of spherical particle scattering obtained theoretically from the Mie code $[6,7]$.

Figure 7 shows the Q-space analysis applied to the three AZRD samples. A constant forward scattering lobe at small q is seen followed by, at larger q, a Guinier regime, a power law regime, and an enhanced backscattering regime. The power law regime yields exponents of $-2.23,-2.17$, and -2.12 for the Ultrafine, Fine and Medium dust samples, respectively; all with uncertainties of \pm 0.05 , hence nearly equivalent. This disclosure of power laws in the scattering by dusts confirms an observation made previously [17].

Thus we find that the Q-space analysis unveils a characteristic description of scattering common to spheres and dusts: a q-independent forward scattering lobe at smallest $q$, followed by a Guinier regime, a power law regime and an enhanced backscattering regime. For AZRD the exponents in the power law regime can discriminate spheres from these particular dusts.

\section{Conclusions.}

We described an experimental system that detects light simultaneously at 31 different angles beginning at $0.32^{\circ}$ to measure all six scattering matrix elements. The scattering matrix elements for irregularly shaped particles behave differently from the ones for spherical particles. Regardless of the shapes of the particles, Guinier analysis indicates particles sizes. Q-space analysis unveils a characteristic description of scattering common to spheres and dusts: a q-independent forward scattering lobe followed by a Guinier regime, a power law regime and an enhanced backscattering regime. Mie scattering from our "quasi-spherical" water droplets shows the power law regime with exponents of -2 and -4 . The exponents of the power laws of the $Q$-space analysis on the Ultrafine, Fine and Medium AZRD samples are $-2.23,-2.17$, and -2.12 , respectively, nearly equivalent within experimental error.

Q-space analysis of scattering for perfect spheres studied under various situations [6-8], show two power law regimes with exponents of -2 and -4 . However, irregularly shaped particles studied so far with Q-space analysis show only one power law with exponents other than -4 . This is based on the work presented here for AZRD, and the analysis in [17]. Currently, we are applying Q-space analysis to the entire Amsterdam data set for irregularly shaped aerosol particles and find single power laws with exponents other than -4 . This work will be presented soon. Therefore, we propose that the power law regime exposed by $Q$-space analysis can discriminate spheres from irregularly shaped particles. With our 
novel experimental apparatus, future work will be applied to test this proposal more broadly and to obtain a theoretical understanding of the origins of these power laws and their exponents.

ACKNOWLEDGEMENTS. The authors wish to thank Tim Sobering, Russell Taylor, and David Huddleston for the detectors electronic design. We are grateful to Russ Reynolds for his help on building and modifying our research instrumentation. Special thanks are given to Justin Maughan for his theoretical support on the Mie code. This work is supported by National Science Foundation under grant no. AGM 1261651 and Army Research Laboratory under grant no. W911NF-14-1-0352.

\section{REFERENCES}

[1] van de Hulst HC. Light scattering by small particles. New York: Dover; 1981.

[2] Bohren CF, Huffman DR. Absorption and Scattering of Light by Small Particles. New York; Wiley; 1983.

[3] Curtis DB, Meland B, Aycibin M, Arnold NP, Grassian VH, Young MA, and Kleiber PD. A laboratory investigation of light scattering from representative components of mineral dust aerosol at a wavelength of $550 \mathrm{~nm}$. J Geophys Res 2008; 113: D08210.

[4] Volten H, Muñoz O, Rol E, de Haan JF, Vassen W, Hovenier JW, Muinonen K, Nousiainen T. Scattering matrices of mineral aerosol particles at $441.6 \mathrm{~nm}$ and $632.8 \mathrm{~nm}$. J Geophys Res 2001; 106: 17375-17401.

[5] Muñoz O, Moreno F, Guirado D, Ramos JL, López A, Girela F, Jerónimo JM, Costillo LP, Bustamante I. Experimental determination of scattering matrices of dust particles at visible wavelengths: The IAA light scattering apparatus. JQSRT 2010; 111: 187-196.

[6] Sorensen CM, Fischbach DJ. Patterns in Mie Scattering. Opt. Commun. 2000; 173; 145-153.

[7] Berg MJ, Sorensen CM, Chakrabarti A. Patterns in Mie Scattering: Evolution when Normalized by the Rayleigh Cross Section. Appl. Opt. 2005; 44: 7487-7493.

[8] Sorensen CM. Q-Space Analysis of Scattering by Particles: A Review. JQSRT 2013: 131: 3-12.

[9] Kuik F, Stammes FP and Hovenier JW. Experimental determination of scattering matrices of water droplets and quartz particles. Appl Opt 1991; 30(33): 4872-4881.

[10] Hunt AJ and Huffman DR. A new polarization-modulated light scattering instrument. Rev. Sci. Instrum 1973; 44: 1753-1762.

[11] Mishchenko MI. Electromagnetic Scattering by Particles and Particle Groups: An Introduction. Cambridge: Cambridge University Press; 2014.

[12] Ferri F, Use of a Charge Coupled Device Camera for Low-Angle Elastic Light Scattering. Rev. Sci. Instrum. 1997; 68: 2265-2273.

[13] Sorensen CM. Light Scattering by Fractal Aggregates: A Review. Aerosol Sci Technol 2001; 35: 648687.

[14] Mokhtari T, Sorensen CM, and Chakrabarti A. Multiple-scattering effects on static light-scattering optical structure factor measurements. Appl Opt 2005; 44(36): 7858-7861

[15] Guinier A, Fournet G, Walker CB, Yudowitch KL. Small Angle Scattering of X-Rays. New York: Wiley; 1955.

[16] Sorensen CM, Shi D. Guinier analysis for homogeneous dielectric spheres of arbitrary size. Opt Commun 2000; 178: 31-36.

[17] Sorensen CM. Q-Space Analysis of Scattering by Dusts. JQSRT 2013; 115: 93-95. 